\title{
Search for di-lepton resonances and Wprimes with CMS
}

\section{D.L. Evans* on behalf of the CMS Collaboration}

University of Bristol, Bristol

E-mail: d.evansecern.ch

\begin{abstract}
The potential of the CMS experiment to discover neutral heavy resonances in the di-electron and di-muon channels as well as a heavy charged boson, $W^{\prime}$, has been studied considering an integrated luminosity of $100 \mathrm{pb}^{-1}$. This integrated luminosity was a realistic expectation of the size of an early LHC dataset circa summer 2008. Assuming Standard Model couplings, discovery of a di-lepton resonance was found to be possible up to a resonance mass of $1.5 \mathrm{TeV}$ and up to approximately $1.2 \mathrm{TeV}$ in the $Z^{\prime} \psi$ model. Massive spin-2 gravitons that arise in the RS model were found to be discoverable in the di-muon channel up to mases of $1.5 \mathrm{TeV}$ assuming a coupling strength $\mathrm{k} / \mathrm{m}_{P l}=0.1$. The discovery of a heavy charged boson, decaying to an electron and antielectron neutrino was found to be possible up to a mass of $2.2 \mathrm{TeV}$. In the absence of a signal, exclusion was found to be possible up to approximately $2.5 \mathrm{TeV}$.
\end{abstract}

Physics at LHC 2008

29 September - October 4, 2008

Split, Croatia

\footnotetext{
* Speaker.
} 


\section{Introduction and Motivation}

In addition to the well-known Standard Model (SM) bosons $g, W^{ \pm}, Z^{0}$ and $\gamma$, many theoretical models predict further neutral heavy gauge bosons, $Z^{\prime}$ or charged heavy gauge bosons, $W^{\prime}$ [1]. Additionally, the extra-dimension model of Randall and Sundrum (RS) predicts massive spin-2 gravitons [2]. The sensitivity of the CMS experiment to such models has been studied.

Neutral resonances can decay to isolated lepton pairs with high $E_{T}$. This signature has been studied in the $e e$ and $\mu \mu$ channels [3, 4]. The $W^{\prime}$ can decay to a lepton and an anti-lepton neutrino, leading to the signature of an isolated lepton with a large balancing missing $E_{T}$ (MET). This signature has been studied in the $e v$ decay channel [5]. Full simulation of the detector response to simulated proton-proton collisions with a centre of mass energy of $14 \mathrm{TeV}$ has been used with an integrated luminosity of $100 \mathrm{pb}^{-1}$. This was considered representative of an early LHC dataset.

\section{Event Selection and Backgrounds}

The single and di-electron selections require identified electrons to pass a standard set of quality and isolation requirements using both the calorimeter and the tracker [3,5]. The di-muon selection requires two muons which are isolated in the tracker [4]. The $W^{\prime}$ event selection uses the ratio of the MET to electron $E_{T}$ as well as the angle between the MET and the electron to further reduce backgrounds [5]. The trigger efficiency is expected to be more than $99 \%$ with respect to the offline selection for signal di-electron events and more than $95 \%$ for single electron events and is more than $97 \%$ for the di-muon selection [3, 4, 5]. The expected di-electron and di-muon mass spectra of a hypothetical signal and expected backgrounds are shown in Figures 1 and 2. The dom-

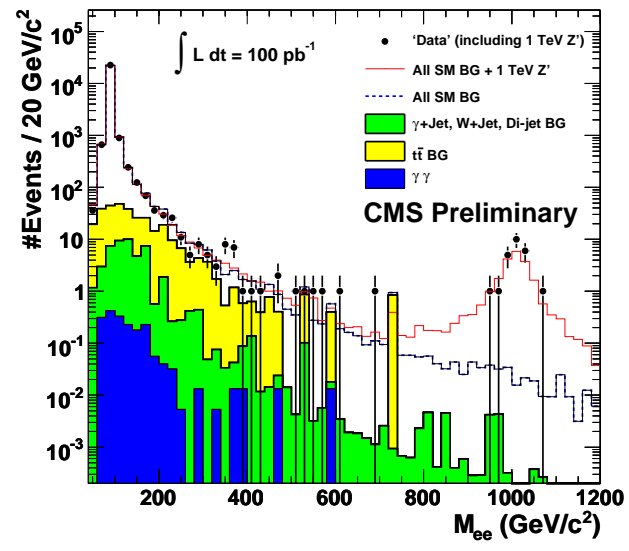

Figure 1: Expected di-electron mass spectrum for backgrounds and a hypothetical signal

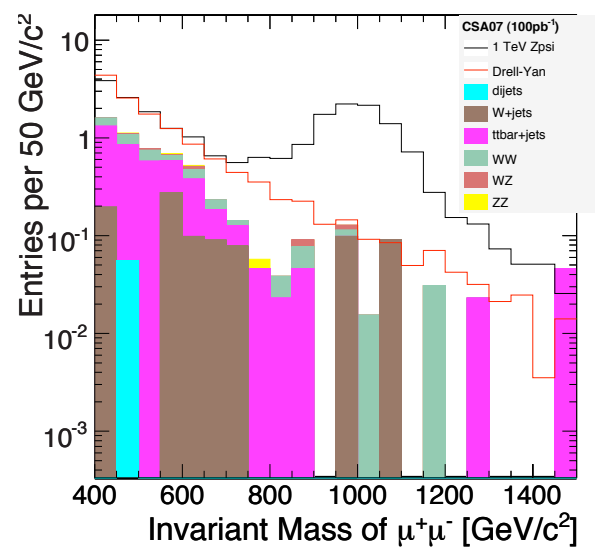

Figure 2: Expected di-muon mass spectrum for backgrounds and a hypothetical signal

inant irreducible background to di-lepton searches at high invariant mass is the Drell-Yan process. This may be determined by normalisation of the simulation to measurements at the $Z^{0}$ pole, or by independent measurement of the Drell-Yan cross section in an intermediate mass control region [3]. The largest irreducible background to $W^{\prime}$ searches is $\mathrm{SM} W^{ \pm}$production. The estimation of this 
background from simulation with appropriate systematics has been studied [5]. The contribution from events containing hadronic jets which are mis-identified can be estimated using the fake rate method. Additionally techniques that exploit the expected charge blind nature of fakes and the selection of control regions through inverting event selection requirements have been studied. The $t \bar{t}$ background, expected to be considerable for all channels discussed, can be estimated by exploiting the $e \mu$ final state or by using $b$-tagging $[3,4,5]$.

\section{Results and Conclusion}

Studies have predicted that neutral heavy gauge bosons, $Z^{\prime}$, with a mass between $1.2 \mathrm{TeV}$ $\left(Z^{\prime} \psi\right)$ and $1.5 \mathrm{TeV}$ (SM couplings) could be discoverable with the di-electon and di-muon channels with $100 \mathrm{pb}^{-1}$ of integrated luminosity. RS gravitons could be discoverable in the di-muon channel for masses up to $1.5 \mathrm{TeV}\left(\mathrm{k} / \mathrm{M}_{P l}=0.1\right)$ [3, 4]. Heavy charged bosons with SM couplings, $W^{\prime}$, could be discoverable up to a mass of $2.2 \mathrm{TeV}$ or excludable up to a mass of $2.5 \mathrm{TeV}$. These results are shown in Figures 3 and 4. It is concluded that new physics could be discovered in a small LHC dataset by exploiting distinctive leptonic final states.

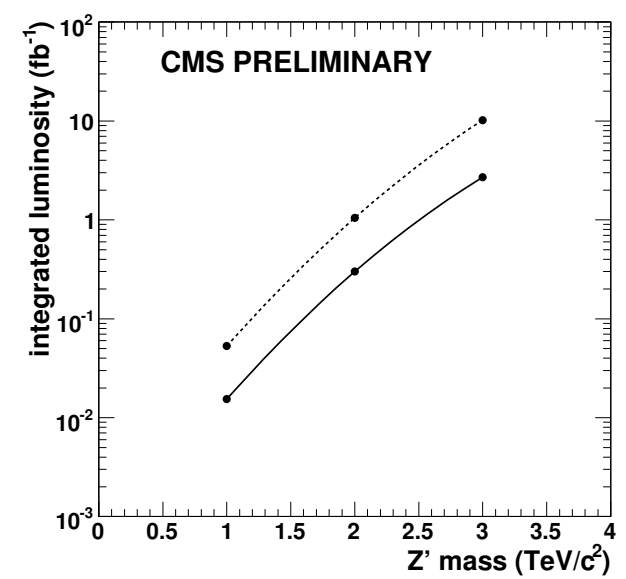

Figure 3: Discovery potential in the dielectron channel ( $Z^{\prime} \psi$ dashed, SSM solid)

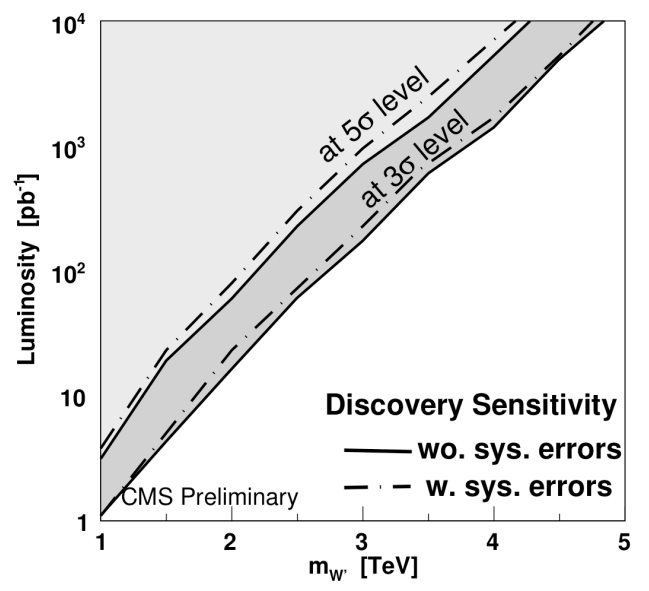

Figure 4: Discovery potential in the electronMET channel ( $W^{\prime}$ with SM couplings)

\section{References}

[1] M. Cvetic and S. Godfrey, Discovery and Identification of Extra Gauge Bosons, arXiv:hep-ph/9504216.

[2] L. Randall and R. Sundrum, A Large Mass Hierarchy from a Small Extra Dimension, Phys. Rev. Lett. 83 (1999) 3370

[3] The CMS Collaboration, Search for High Mass Resonance Production Decaying into an Electron Pair in the CMS Experiment, CMS PAS-EXO-08-001

[4] The CMS Collaboration, Search for New High-Mass Resonances Decaying to Muon Pairs in the CMS Experiment, CMS PAS-SBM-07-002

[5] The CMS Collaboration, Discovery Potential of $W^{\prime} \rightarrow e v$ at CMS, CMS PAS-EXO-08-004 